\title{
Measurements, Modeling and Simulation of the Off-Body Radio Channel for the Implementation of Bodyworn Antenna Diversity at $868 \mathrm{MHz}$
}

\author{
Simon L. Cotton, Member, IEEE, and William G. Scanlon, Member, IEEE
}

\begin{abstract}
This paper presents a systematic measurement campaign of diversity reception techniques for use in multiple-antenna wearable systems operating at $868 \mathrm{MHz}$. The experiments were performed using six time-synchronized bodyworn receivers and considered mobile off-body communications in an anechoic chamber, open office area and a hallway. The cross-correlation coefficient between the signal fading measured by bodyworn receivers was dependent upon the local environment and typically below 0.7. All received signal envelopes were combined in post-processing to study the potential benefits of implementing receiver diversity based upon selection combination, equal-gain and maximal-ratio combining. It is shown that, in an open office area, the $5.7 \mathrm{~dB}$ diversity gain obtained using a dual-branch bodyworn maximal-ratio diversity system may be further improved to 11.1 dB if a six-branch system was used. First- and second-order theoretical equations for diversity reception techniques operating in Nakagami fading conditions were used to model the postdetection combined envelopes. Maximum likelihood estimates of the Nakagami- $m$ parameter suggest that the fading conditions encountered in this study were generally less severe than Rayleigh. The paper also describes an algorithm that may be used to simulate the measured output of an $M$-branch diversity combiner operating in independent and identically-distributed Nakagami fading environments.
\end{abstract}

Index Terms-Diversity methods, fading channels, propagation, propagation measurements, radio propagation, UHF communication.

\section{INTRODUCTION}

I NDOOR mobile bodyworn communications systems are often subjected to complicated, time-varying radio channel conditions. For off-body communications, where a bodyworn radio terminal communicates with a local transceiver such as an access point or indoor cellular base station situated on a nearby wall, the multipath fading experienced on these channels can be significant and may severely deteriorate the quality of the radio link [1]. At frequencies in the range $800-915 \mathrm{MHz}$, the presence of the human body can also introduce complex antenna-

Manuscript received December 16, 2008; revised May 14, 2009. First published October 02, 2009; current version published December 01, 2009.

The authors are with School of Electronics, Electrical Engineering and Computer Science, Institute of Electronics, Communications and Information Technology, The Queen's University of Belfast, Belfast BT3 9DT, U.K. (e-mail: simon.cotton@qub.ac.uk; w.scanlon@qub.ac.uk).

Color versions of one or more of the figures in this paper are available online at http://ieeexplore.ieee.org.

Digital Object Identifier 10.1109/TAP.2009.2033439 body interaction effects. These include near-field coupling, radiation pattern fragmentation and shifts in antenna impedance [2], [3], which may degrade the efficiency of the bodyworn system and reduce signal reliability. One method of providing mitigation of these deleterious effects is by employing diversity at the receiver. Space, time, frequency and polarization diversity methods are often utilized with the aim of providing partially decorrelated signal versions at the receiver, which may provide some mitigation of the effects of fading. If the diversity branches are suitably uncorrelated and have comparable mean signal levels, then it is expected that the combination of these signals will have a higher signal-to-noise ratio (SNR) than if one branch is considered in isolation [4].

Signal variation in mobile fading channels is often reported using a combination of first-order statistics such as probability density functions (pdfs) and cumulative distribution functions (CDFs), and second-order statistics such as level crossing rate (LCR) and average fade duration (AFD). Second-order statistics are of particular interest when designing mobile radio systems [5] as they give a direct indication as to the rate of change, with respect to time, of the wireless channel. Among their uses is the design of error correcting codes, optimization of interleaver size and system throughput analysis as well as channel modeling. A pdf that has recently been used to model fading on channels that incorporate the human body [1], [6]-[8] is the Nakagami- $m$ pdf [9]. Moreover, it has been used in the analysis of diversity reception techniques [10]-[13] for a number of decades. The Nakagami pdf has proven popular due to its ability to approximate the Rice pdf [14] for $m>1$, reduce to the Rayleigh pdf $(m=1)$ and describe fading conditions that are worse than Rayleigh $(m<1)$. Another important feature of the Nakagami distribution is that it does not place the restriction of uniform magnitude for scattered vectors as observed in the theoretical Rice and Rayleigh fading models. Analytical second-order expressions for diversity reception techniques in Nakagami fading are readily available [15], [16]. A number of proposed simulation techniques also exist for simulating waveforms [17]-[19] and decision errors seen at the output of the demodulator [20] in Nakagami fading conditions. Zhang's [17] approach to formulating Nakagami random variables (RVs) was to generate Gamma RVs from correlated Gaussian RVs by applying either Cholesky decomposition or the technique of autoregressive modeling. As the Gamma distribution is the square of the Nakagami distribution, the mapping from Gamma to Nakagami RV is straightforward. Taking a different approach, Beaulieu's [18] Nakagami simulator creates 
Nakagami RVs using a two-step transformation process from time-correlated Rayleigh RVs. This method is attractive in that autocorrelated Rayleigh RVs can easily be generated using commercially available software such as Matlab. One drawback of this technique is that it may require an extra interpolation stage for non-standard values of the Nakagami- $m$ parameter. More recently, Filho et al. [19] have proposed a rank matching approach that also produces autocorrelated Nakagami RVs from time-correlated Rayleigh RVs while taking advantage of the Nakagami-Gamma relationship mentioned above. It is this methodology that shall be adopted here and further expanded in Section V.

At UHF frequencies, wearable multiple antenna systems have been considered for military applications such as the Joint Tactical Radio System and the Joint Threat Warning System [21]. Other possible civilian uses include wearable wireless personal area networks [1] and remote patient monitoring [22]. Mobile off-body measurements were presented in [23] where only one type of diversity combination technique was considered and we did not isolate the effects of multipath generated by the environment or report diversity gain results. More recently, channel measurement campaigns were conducted that aimed to assess the effectiveness of bodyworn antenna diversity for on-body [24]-[26] and body-to-body [27], [28] links. In [24], it was shown that for on-body communications, diversity reception was most effective when the on-body path between transmitter and diversity receiver crossed from the front to the back of the human body and the user was mobile in a multipath environment. Selection combination [27], [28], equal-gain and maximal-ratio combining [27] techniques have all been investigated for the purpose of combating body-shadowing and channel impairments in body-to-body communications channels. To the best of our knowledge cross-correlation coefficients relating to the signal fading experienced at various positions on the upper human body based on time synchronous measured data for offbody channels (i.e., body to fixed access point), have yet to be published in the open literature. Furthermore, a statistical characterization of dual-branch bodyworn diversity through experimentation, modeling and simulation is presented.

This paper is organized as follows. Section II describes the bodyworn diversity measurement system, indoor environments and experimental procedure. Cross-correlation coefficients between variations of the fading signals experienced by potential diversity branch pairs located on the upper human body are presented in Section III. In Section IV, diversity gain results are reported for a number of bodyworn configurations. The theoretical second-order diversity equations used to model all experimental data are presented in Section V alongside the modeling and simulation of a selection of diversity-combined envelopes. Finally, Section VI presents the conclusions.

\section{EXPERIMENTAL PROCEDURE}

\section{A. Bodyworn Array}

The bodyworn diversity measurement system consisted of a fixed transmitter and an array of six bodyworn self-contained data logging $868 \mathrm{MHz}$ receivers (Fig. 1). The transmitter
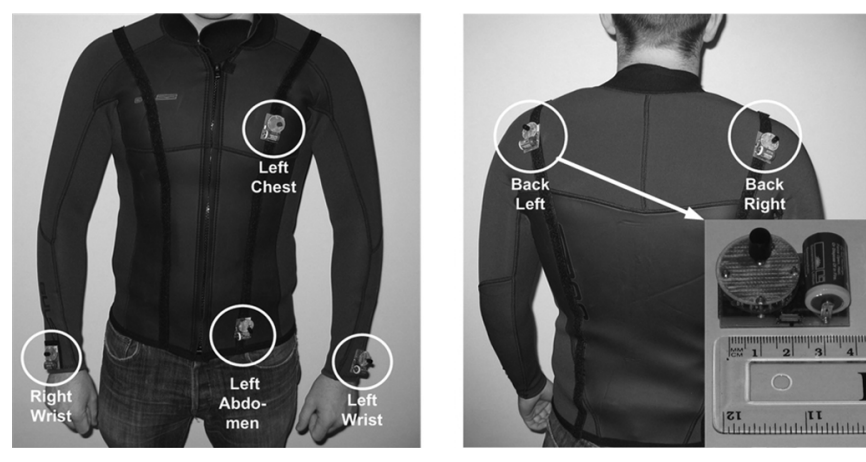

Fig. 1. Bodyworn measurement system with receivers at 6 locations.

consisted of a NovaSource ${ }^{1}$ G6 RF signal source configured to deliver $0 \mathrm{dBm}$ of continuous wave through a vertically polarized $\lambda / 4$ stub antenna. Each of the receivers in the wearable arrangement was a pre-calibrated Crossbow ${ }^{2}$ Mica2Dot wireless sensor with a modified 3.6 V, 0.95 Ah lithium-thionyl chloride power supply unit and a normal-mode helical antenna mounted on a $25.4 \mathrm{~mm}$ diameter circular ground plane (Fig. 1). A 10-bit on-board analogue to digital converter was used to quantize the received signal voltage from the sensor's Chipcon ${ }^{3}$ CC1000 RF chip. The sensors were attached to a $3 \mathrm{~mm}$ thick, $80 \%$ polychloroprene/20\% nylon contoured jacket with sewn on mounting pads. The jacket was tight fitting to minimize spurious antenna-body separation effects. As shown in Fig. 1, the signal voltage logging sensors were placed at six body locations: back-left, back-right, left-abdomen, left-chest and one on each of the user's wrists, all referenced from the test subject's perspective.

\section{B. Indoor Environments}

Measurements were taken in an anechoic chamber $\left(54 \mathrm{~m}^{2}\right)$, an open office area $\left(244.2 \mathrm{~m}^{2}\right)$ and a hallway $\left(13.5 \mathrm{~m}^{2}\right)$ on the ground floor of the ECIT building at Queen's University, Belfast, UK. Fig. 2 shows a plan of the measurement environment in relation to the rest of the ground floor. The building is of recent construction, consisting mainly of metal studded dry wall with a metal tiled floor covered with polypropylene-fiber, rubber backed carpet tiles, and a metal ceiling with mineral fiber tiles and recessed louvered luminaries suspended $2.7 \mathrm{~m}$ above floor level. The open area office contained a number of soft partitions, PCs, chairs and desks constructed from medium density fiberboard. The anechoic chamber was housed in conductive shielding and lined with pyramidal RF absorbers.

\section{Measurement Procedure}

The receiver array was worn by an adult male of mass $83 \mathrm{~kg}$ and height $1.72 \mathrm{~m}$. The transmitter was attached to a wooden telescopic pole at a height of $2.45 \mathrm{~m}$ above floor level. All three environments were unoccupied for the duration of the measurements. Each sensor was activated to make time synchronized

\footnotetext{
${ }^{1}$ http://www.nova-eng.com/ (12/09/2008)

${ }^{2} \mathrm{http}: / /$ www.xbow.com/ (12/09/2008)

${ }^{3}$ http://www.ti.com/ (12/09/2008)
} 


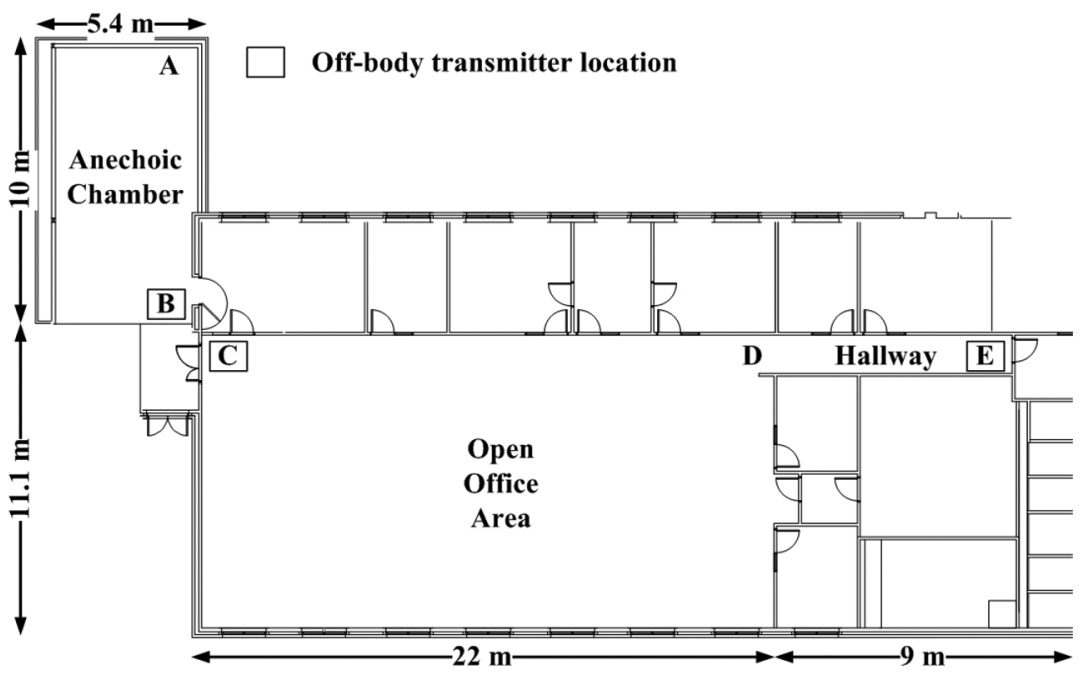

Fig. 2. Plan view of measurement locations showing start and stop positions also transmitter locations.

TABLE I

NOISE FLOOR (NF), STANDARD DEVIATION (STD.) AND WEAKEST RECORDED SAMPLE (WRS) MEASUREMENTS

\begin{tabular}{|c|c|c|c|}
\hline Environment & NF (dBm) & STD. $(\mathrm{dB})$ & WRS $(\mathrm{dBm})$ \\
\hline Anechoic Chamber & -94.4 & 3.1 & -86.1 \\
\hline Open Office Area & -93.1 & 2.9 & -90.0 \\
\hline Hallway & -93.2 & 2.6 & -89.9 \\
\hline
\end{tabular}

recordings of the signal voltage at $3.91 \mathrm{~ms}$ intervals (256 samples/s). As the units were based upon wireless sensors, time synchronization was effected using base station software, and is accurate to within one sample period. User trajectories are denoted by the order of the letters for the respective points between which the journey is performed; for paths $\mathrm{AB}$ (anechoic chamber), CD (open office area) and DE (hallway) the TX was fixed at positions B, C and E, respectively (Fig. 2). The use of six bodyworn receivers in these experiments meant that fifteen unique receiver pairings were available for post-combining over each of the individual path trajectories. The entire measurement session consisted of three consecutive repeated trials of each scenario yielding a total of 211800 signal voltage samples. Table I shows the mean recorded noise floor and corresponding weakest recorded sample observed in each environment. For all forward journeys (AB, DC and $\mathrm{DE})$ the frontal body receivers were positioned in line of sight (LOS) with the back receivers in non-line of sight (NLOS). The corresponding return journeys (BA, CD and ED) allowed a reverse analysis of LOS conditions. Average straight line velocity for each of the mobile sessions was observed to be approximately $1.0 \mathrm{~m} / \mathrm{s}$.

\section{CROSS-CORRELATION}

To prepare the measurement data for the calculation of the cross-correlation coefficient $\left(\rho_{C}\right)$ between the signal fading measured by each receiver, the raw signal envelopes were normalized by removing the local mean over an averaging window of length $\sim 4.5 \lambda$, which was estimated from the raw measurement data. The smallest data set considered for the calculation of $\rho_{C}$ consisted of 1200 samples for the path ED in the hallway environment. This was also the smallest data set used for the calculation of diversity gain (Section IV) and parameter estimation (Section V). For a diversity scheme to be effective, each antenna element should receive statistically independent versions of the transmitted signal, significantly reducing the likelihood that all branches are experiencing simultaneous fading. As a relative figure of merit [29], two signals are said to be suitably de-correlated if their cross-correlation coefficient is less than 0.7. The cross-correlation coefficient between the signal fading recorded by each of the bodyworn receivers was estimated using (1) from [25]. The mean cross-correlation coefficient, $\overline{\rho_{C}}$, was then calculated by taking the average of $\rho_{C}$ from the three trials for a particular path. The results of these calculations for the anechoic chamber and open office area environments are provided in Tables II and III alongside an estimate of the standard error (SE).

The highest mean cross-correlation coefficients were found to occur while the test subject moved along paths $\mathrm{AB}$ and $\mathrm{BA}$ in the anechoic chamber. For this environment, $\overline{\rho_{C}}$ ranged from -0.23 for the back-right/left-wrist positioned receivers to 0.82 for the back-right/left-chest receivers (path $\mathrm{AB}$ ) and -0.11 for the left-chest/right-wrist pair to 0.96 for back-left/back-right receivers (path BA) with median $\overline{\rho_{C}}$ of 0.00 for $\mathrm{AB}$ and 0.39 for BA. When the same experiments were conducted in the open office area and hallway environments, an increase in the multipath generated by the local surroundings was found to have a noticeable effect upon the cross-correlation of signal fading measured by each of the bodyworn receivers. In the open office area (Table III), the estimates of $\overline{\rho_{C}}$ were over the reduced ranges of -0.09 to 0.29 (median 0.14 ) for the path DC and -0.11 to 0.51 (median 0.08) for the path $\mathrm{CD}$. A similar trend was observed for the hallway environment, where $\overline{\rho_{C}}$ ranged from -0.17 to 0.30 (median 0.05) for path DE and -0.22 to 0.37 (median 0.02) for path ED. While the estimated $\overline{\rho_{C}}$ values for the anechoic chamber were larger than those for corresponding receiver pairs in the open office and hallway environments, they were nonetheless typically lower than 0.7 , with only 6 receiver pairs out of a 
TABLE II

Mean Cross-Correlation Coefficient, MRC Diversity Gain and DIMSL Alongside the Estimated STANDARD ERROR (SE) FOR THE THREE EXPERIMENTAL TRIALS CONDUCTED IN THE ANECHOIC CHAMBER

\begin{tabular}{|c|c|c|c|c|c|c|c|c|c|c|c|c|}
\hline \multirow[b]{3}{*}{ Receivers } & \multicolumn{4}{|c|}{ Correlation } & \multicolumn{4}{|c|}{$\operatorname{MRC}(\mathrm{dB})$} & \multicolumn{4}{|c|}{ DIMSL (dB) } \\
\hline & \multicolumn{2}{|c|}{ Path AB } & \multicolumn{2}{|c|}{ Path BA } & \multicolumn{2}{|c|}{ Path AB } & \multicolumn{2}{|c|}{ Path BA } & \multicolumn{2}{|c|}{ Path AB } & \multicolumn{2}{|c|}{ Path BA } \\
\hline & $\rho_{C}$ & SE & $\rho_{C}$ & SE & MRC & SE & MRC & SE & $\overline{\text { DIMSL }}$ & SE & $\overline{\text { DIMSL }}$ & SE \\
\hline Back-Left / Back-Right & 0.77 & 0.02 & 0.96 & 0.00 & 1.0 & -28.6 & 1.6 & -36.4 & 3.6 & -25.9 & 0.3 & -48.9 \\
\hline $\begin{array}{l}\text { Back-Left / Left } \\
\text { Abdomen }\end{array}$ & 0.44 & 0.07 & 0.38 & 0.03 & 4.1 & -44.1 & 0.4 & -14.0 & 0.7 & -30.3 & 13.4 & -39.3 \\
\hline Back-Left / Left-Chest & 0.76 & 0.03 & 0.80 & 0.01 & 6.3 & -25.6 & 2.1 & -6.4 & 0.3 & -34.5 & 4.9 & -24.7 \\
\hline Back-Left / Left-Wrist & -0.06 & 0.09 & 0.29 & 0.07 & 5.1 & -44.3 & 0.4 & -15.0 & 3.5 & -25.3 & 14.6 & -11.6 \\
\hline Back-Left / Right-Wrist & -0.21 & 0.03 & -0.03 & 0.03 & 6.8 & -36.8 & 0.4 & -10.1 & 0.7 & -25.9 & 13.6 & -21.3 \\
\hline $\begin{array}{l}\text { Back-Right / Left- } \\
\text { Abdomen }\end{array}$ & 0.65 & 0.06 & 0.44 & 0.04 & 1.7 & -26.9 & 1.2 & -41.6 & 3.1 & -36.7 & 13.1 & -31.9 \\
\hline Back-Right / Left-Chest & 0.82 & 0.05 & 0.89 & 0.00 & 1.2 & -17.6 & 4.1 & -26.3 & 3.7 & -35.0 & 4.5 & -24.7 \\
\hline Back-Right / Left-Wrist & -0.23 & 0.09 & 0.39 & 0.07 & 1.8 & -32.8 & 1.1 & -28.0 & 7.1 & -28.7 & 14.3 & -11.7 \\
\hline Back-Right / Right-Wrist & 0.00 & 0.04 & -0.05 & 0.02 & 4.3 & -29.4 & 1.0 & -26.5 & 2.9 & -31.9 & 13.3 & -21.8 \\
\hline $\begin{array}{l}\text { Left-Abdomen / Left- } \\
\text { Chest }\end{array}$ & 0.52 & 0.16 & 0.52 & 0.08 & 2.9 & -36.0 & 0.7 & -22.5 & 0.7 & -34.7 & 8.6 & -21.1 \\
\hline $\begin{array}{l}\text { Left-Abdomen / Left- } \\
\text { Wrist }\end{array}$ & -0.18 & 0.13 & 0.45 & 0.04 & 4.4 & -25.1 & 4.3 & -26.2 & 4.0 & -32.8 & 1.2 & -24.9 \\
\hline $\begin{array}{l}\text { Left-Abdomen / Right- } \\
\text { Wrist }\end{array}$ & 0.26 & 0.06 & 0.11 & 0.01 & 7.3 & -30.6 & 3.6 & -28.0 & 0.3 & -41.6 & 0.2 & -35.3 \\
\hline Left-Chest / Left-Wrist & -0.21 & 0.04 & 0.47 & 0.06 & 7.2 & -39.2 & 0.9 & -6.1 & 3.4 & -35.7 & 9.7 & -26.4 \\
\hline Left-Chest / Right-Wrist & -0.05 & 0.07 & -0.11 & 0.03 & 6.6 & -45.6 & 0.8 & -12.3 & 0.8 & -29.7 & 8.8 & -24.8 \\
\hline Left-Wrist / Right-Wrist & -0.11 & 0.01 & 0.00 & 0.06 & 2.0 & -18.6 & 4.6 & -34.5 & 4.2 & -25.2 & 1.0 & -28.2 \\
\hline
\end{tabular}

possible 30 (15 per path, over $\mathrm{AB}$ and $\mathrm{BA}$ ) being in excess of this figure (Table II).

\section{Diversity GAIN AND DifFerence IN MEAN Signal LEVEL}

Three diversity-combining techniques were considered: selection, maximal-ratio and equal-gain combining. Selection combination (SC) is a form of switched diversity that provides an output by selecting the branch having the highest input SNR, although in practice the branch with the largest signal plus noise contribution is used due to the difficulty in measuring SNR [29]. Thus for a diversity array consisting of $M$ branches, the SC output level $R$ is

$$
R=\max \left(r_{1}, r_{2}, \ldots, r_{M}\right)
$$

where $r_{M}$ is the signal level observed in the $M^{\text {th }}$ branch of the diversity receiver. In postdetection maximal-ratio combining (MRC), instantaneous signals in each of the $M$ branches of the receiver are weighted with respect to their SNR before being summed. According to [30], if it is assumed that the noise in each channel is independent of the signal, the signals measured by each branch are locally coherent and the noise components are locally incoherent (i.e., uncorrelated) then it can be shown that the output of an $M$-branch postdetection maximal-ratio combiner is equal to the sum of the individual power ratios [30, Eq. (13)]. Following [30], if we assume that the local noise power is unity then the signal output of an $M$-branch maximal-ratio combiner may be written as

$$
R=\sqrt{r_{1}^{2}+r_{2}^{2}+\ldots+r_{M}^{2}} .
$$

The complexity of producing weighted coefficients in MRC may be reduced by using postdetection equal-gain combining (EGC) where all weighting factors are assumed constant. If it assumed that the noise power is equal to unity on all branches [30] then the equation for the signal measured at the output of an $M$-branch equal-gain combiner may be obtained from [30, Eq. (36)] and expressed as

$$
R=\frac{r_{1}+r_{2}+\ldots+r_{M}}{\sqrt{M}} .
$$

An important concept for diversity systems is diversity gain. Turkmani et al. [31] provide a definition of diversity gain as the difference in signal level of the branch with the highest mean and that of the output of the diversity combiner for a given probability or signal reliability. Diversity gain is dependent on the cross-correlation and power imbalance between branches [31]. All diversity gain calculations in this paper were made at a signal reliability of $90 \%$ (a cumulative probability of 0.1 ).

In Section IV, all calculations of the diversity gain and the difference in mean signal level (DIMSL) between branches were computed using the raw signal voltage envelope. The results from each of the three trials for a particular receiver pair and 
TABLE III

Mean Cross-Correlation CoefFicient, MRC Diversity Gain and DIMSL Alongside the Estimated STANDARD ERROR (SE) FOR THE THREE EXPERIMENTAL TRIALS CONDUCTED IN THE OPEN OFFICE AREA

\begin{tabular}{|c|c|c|c|c|c|c|c|c|c|c|c|c|}
\hline & \multicolumn{4}{|c|}{ Correlation } & \multicolumn{4}{|c|}{ MRC (dB) } & \multicolumn{4}{|c|}{ DIMSL (dB) } \\
\hline & \multicolumn{2}{|c|}{ Path DC } & \multicolumn{2}{|c|}{ Path CD } & \multicolumn{2}{|c|}{ Path DC } & \multicolumn{2}{|c|}{ Path CD } & \multicolumn{2}{|c|}{ Path DC } & \multicolumn{2}{|c|}{ Path CD } \\
\hline Receivers & $\overline{\rho_{C}}$ & $\mathrm{SE}$ & $\overline{\rho_{C}}$ & $\mathrm{SE}$ & $\overline{\mathrm{MRC}}$ & SE & $\overline{\mathrm{MRC}}$ & SE & DIMSL & SE & $\overline{\text { DIMSL }}$ & SE \\
\hline Back-Left / Back-Right & 0.28 & 0.10 & 0.15 & 0.09 & 4.6 & -17.2 & 5.1 & -19.8 & 2.8 & -14.9 & 3.0 & -16.6 \\
\hline $\begin{array}{l}\text { Back-Left / Left } \\
\text { Abdomen }\end{array}$ & 0.16 & 0.07 & 0.14 & 0.06 & 5.8 & -31.0 & 3.6 & -27.0 & 1.2 & -24.0 & 6.3 & -44.5 \\
\hline Back-Left / Left-Chest & 0.29 & 0.09 & 0.51 & 0.04 & 2.7 & -26.1 & 5.3 & -34.1 & 2.3 & -28.5 & 3.6 & -31.6 \\
\hline Back-Left / Left-Wrist & 0.14 & 0.05 & 0.08 & 0.10 & 3.2 & -22.5 & 4.3 & -20.2 & 1.5 & -23.5 & 4.9 & -23.6 \\
\hline Back-Left / Right-Wrist & -0.03 & 0.06 & 0.08 & 0.08 & 5.4 & -22.9 & 4.8 & -20.7 & 1.1 & -24.6 & 3.7 & -30.3 \\
\hline $\begin{array}{l}\text { Back-Right / Left- } \\
\text { Abdomen }\end{array}$ & 0.14 & 0.16 & -0.06 & 0.08 & 5.4 & -23.3 & 6.3 & -13.7 & 1.8 & -20.3 & 3.4 & -16.9 \\
\hline Back-Right / Left-Chest & 0.18 & 0.15 & -0.05 & 0.08 & 2.2 & -6.0 & 8.1 & -27.3 & 5.1 & -13.3 & 1.5 & -33.8 \\
\hline Back-Right / Left-Wrist & 0.07 & 0.05 & -0.11 & 0.07 & 2.7 & -18.1 & 6.9 & -19.4 & 4.5 & -11.0 & 2.3 & -16.6 \\
\hline Back-Right / Right-Wrist & -0.03 & 0.06 & 0.14 & 0.10 & 4.0 & -13.9 & 7.4 & -21.7 & 2.6 & -18.2 & 1.2 & -27.7 \\
\hline $\begin{array}{l}\text { Left-Abdomen / Left- } \\
\text { Chest }\end{array}$ & 0.16 & 0.09 & 0.05 & 0.02 & 2.7 & -24.0 & 3.9 & -27.8 & 3.3 & -17.8 & 2.7 & -33.3 \\
\hline $\begin{array}{l}\text { Left-Abdomen / Left- } \\
\text { Wrist }\end{array}$ & 0.18 & 0.04 & 0.25 & 0.04 & 3.3 & -15.3 & 5.3 & -19.7 & 2.6 & -14.9 & 1.4 & -27.0 \\
\hline $\begin{array}{l}\text { Left-Abdomen / Right- } \\
\text { Wrist }\end{array}$ & 0.02 & 0.03 & 0.06 & 0.12 & 4.9 & -12.9 & 5.4 & -30.7 & 1.3 & -22.5 & 2.6 & -31.1 \\
\hline Left-Chest / Left-Wrist & -0.09 & 0.07 & 0.18 & 0.11 & 4.7 & -25.7 & 3.6 & -20.2 & 0.8 & -23.4 & 1.4 & -28.2 \\
\hline Left-Chest / Right-Wrist & 0.05 & 0.06 & 0.04 & 0.03 & 2.7 & -10.2 & 7.1 & -34.9 & 2.5 & -19.5 & 0.4 & -33.2 \\
\hline Left-Wrist / Right-Wrist & 0.12 & 0.01 & -0.10 & 0.03 & 4.5 & -25.2 & 7.0 & -10.8 & 2.1 & -18.0 & 1.3 & -24.0 \\
\hline
\end{tabular}

path were then averaged to estimate the mean diversity gain and mean DIMSL ( $\overline{\text { DIMSL }}$. Tables II and III show the complete $\overline{\text { DIMSL }}$ results for the anechoic chamber and open office area, alongside the standard error expressed in $\mathrm{dB}$. As both of these tables show, the $\overline{\text { DIMSL }}$ was dependent on the whether the test subject was moving towards or away from the transmitter and the physical composition of the local surroundings. When the test subject moved towards the transmitter in the anechoic chamber (path $\mathrm{AB}$ ), the $\overline{\mathrm{DIMSL}}$ ranged from 0.3 to $7.1 \mathrm{~dB}$ (median $3.1 \mathrm{~dB}$ ), the corresponding ranges for the open office area (path DC) and hallway (path DE) were 0.8 to $5.1 \mathrm{~dB}$ (median $2.3 \mathrm{~dB}$ ) and 0.5 to $3.9 \mathrm{~dB}$ (median $2.7 \mathrm{~dB}$ ), respectively. An increase in the multipath contribution caused by the reflection, diffraction and scattering of the transmitted electromagnetic waves from the upright, ceiling and floor structures in the open office area and hallway tended to reduce the magnitude of DIMSL obtained in the anechoic chamber as the test subject moved away from the transmitter (Tables II and III).

Using (1)to (3), the raw signal envelopes recorded by each of the wireless sensors were combined in post-processing to form virtual postdetection [30] dual-branch diversity receivers. The diversity gain for each receiver pairing was individually estimated for each of the three trials, then averaged to calculate the mean diversity gain for selection combination $(\overline{\mathrm{SC}})$, equal-gain $(\overline{\mathrm{EGC}})$ and maximal-ratio $(\overline{\mathrm{MRC}})$ combining for that particular receiver pair and path. Maximal-ratio combining has the property that among all of the systems considered here, it yields the maximum SNR of the output signal [30]. Tables II and III present the estimated $\overline{\mathrm{MRC}}$ diversity gain that would be ob- tained, if a postdetection dual-branch bodyworn system was implemented using the designated antenna positions. The standard error of the estimated maximal-ratio diversity gain for the three trials is also provided in Tables II and III and is expressed in $\mathrm{dB}$. In the anechoic chamber, the $\overline{\mathrm{MRC}}$ ranged from 1.0 to $7.3 \mathrm{~dB}$ with a median value of $4.3 \mathrm{~dB}$ for the path $\mathrm{AB}$, and $0.4 \mathrm{~dB}$ to $4.6 \mathrm{~dB}$, median $1.1 \mathrm{~dB}$ for the path $\mathrm{BA}$. The reduction in $\overline{\mathrm{MRC}}$ diversity gain for path BA was most likely caused by the much larger DIMSL observed for the majority of receiver pairs in this scenario (Table II). The $\overline{\mathrm{MRC}}$ diversity gain for path DC in the open office area was over the slightly improved range of 2.2 to $5.8 \mathrm{~dB}$, albeit with a reduced median of $4.0 \mathrm{~dB}$ when compared to the anechoic chamber. However, when compared to path BA in the anechoic chamber, the equivalent path $\mathrm{CD}$ in the open office area had significantly improved $\overline{\mathrm{MRC}}$ diversity gains in the range $3.6 \mathrm{~dB}$ to $8.1 \mathrm{~dB}$ with median $5.3 \mathrm{~dB}$. The reduced dimensions of the hallway helped to provide comparable $\overline{\mathrm{MRC}}$ diversity gain for both travelling towards ( 2.5 to $8.2 \mathrm{~dB}$, median $6.2 \mathrm{~dB}$ ) and away (2.6 dB to $8.2 \mathrm{~dB}$, median $4.7 \mathrm{~dB}$ ) from the transmitter.

To illustrate the advantage of having more than two available diversity branches, we initially consider a two-branch bodyworn system consisting of receivers placed at the back-left and leftchest positions for the path $\mathrm{CD}$ in the open office area, and then a six-branch system using all of the receivers accessible in this scenario. For this particular path, the back-left receiver recorded the signal envelope with the highest overall mean signal level out of the six on-body positions. When paired with the left-chest receiver, the $\overline{\rho_{C}}$ between the signal fading recorded by these 


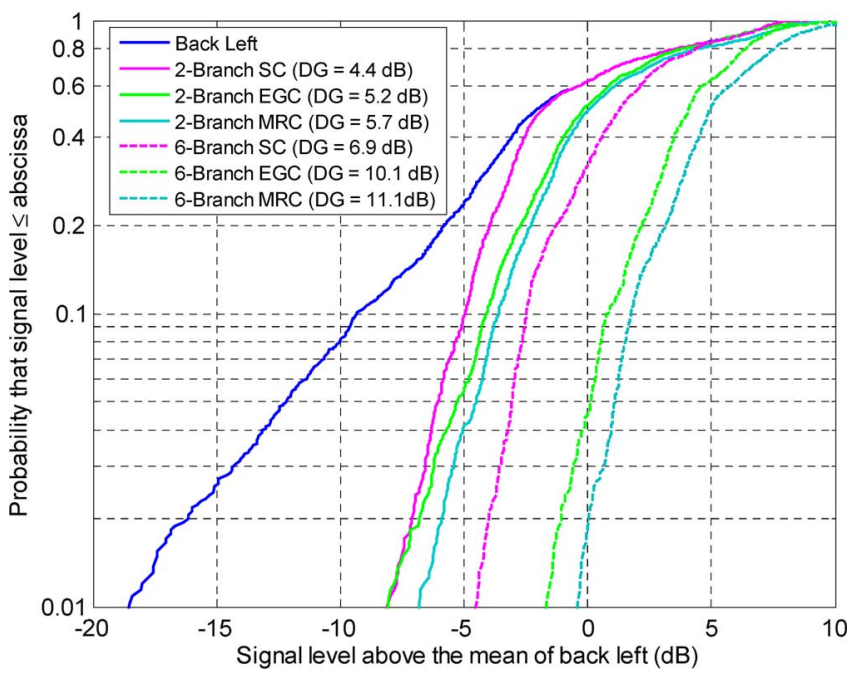

Fig. 3. Cumulative distribution functions for hypothetical two-branch (backleft/left-chest) and six-branch bodyworn spatial diversity systems operating in open office area (path $\mathrm{CD}$ ), showing diversity gain improvement compared to mean level of back-left receiver (branch with highest mean signal level). Note diversity gain (DG) values given in brackets.

two receivers was 0.51 with an $\overline{\mathrm{DIMSL}}$ of $3.6 \mathrm{~dB}$. Consistent with the analysis technique of Turkmani [31], Fig. 3 shows the $\mathrm{CDF}$ of the signal envelopes from trial 2 for the back-left and all three combining techniques relative to the mean signal level of the branch with the highest average power. Note that for trial 2 (Fig. 3), the estimated two-branch MRC diversity gain (5.7 dB) for this receiver pairing was marginally above the $\overline{\mathrm{MRC}}$ diversity gain given in Table III ( $5.3 \mathrm{~dB})$. By increasing the number of receive antennas from two to six, significant diversity gain can be achieved for all three combining techniques, with both MRC and EGC ensuring little fading below the mean signal level of the back-left receiver (Fig. 3). As expected, MRC offered the highest diversity gain of $11.1 \mathrm{~dB}$, which was vastly superior to the two-branch case.

\section{Modeling of Diversity COMBINED ENVELopes OPERATING IN NAKAGAMI FADING}

\section{A. SC, MRC and EGC Diversity Reception in Nakagami Fading}

Building on the results presented in Section IV which have shown that, for most scenarios where the user is expected to be mobile, a worthwhile improvement in radio system performance may be obtained by providing more than one signal branch in bodyworn systems. We now use first- and second-order equations for diversity reception techniques operating in Nakagami fading conditions to characterize the distribution of small-scale fading experienced in bodyworn diversity systems. This analysis was chosen based on previous work that has shown that the fading experienced by bodyworn receivers placed on the upper torso and limbs of a mobile user in an indoor environment may be adequately described by Nakagami first- and second-order statistics [1]. Under the assumption of identical Nakagami fading conditions, equal noise power and independence between diversity branches, closed-form formulae for the CDFs of SC and MRC in the presence of Nakagami distributed fading are presented in [15]. The level crossing rate of the diversity-combined envelope may be defined as the number of positive (or negative) transitions beyond a threshold level $R$ in a given amount of time. The LCR $\left(N_{R}\right)$ is expressed analytically in terms of the time derivative $\dot{r}$ of the combined envelope $r$ and the joint probability of $\dot{r}$ and $r$ at $r=R$

$$
N_{R}=\int_{0}^{\infty} \dot{r} p(\dot{r}, r=R) d \dot{r}
$$

For a selection combining scheme with $M$ diversity branches operating in Nakagami fading conditions, the LCR $\left(N_{R}\right)$, normalized to the maximum Doppler frequency $f_{m}$, may be represented by [15]

$$
\frac{N_{R}}{f_{m}}=\frac{\sqrt{2 \pi} M \Gamma^{M-1}\left(m, m \rho^{2}\right)}{\Gamma^{M}(m)}\left(m \rho^{2}\right)^{m-0.5} \exp \left(-m \rho^{2}\right)
$$

where $m$ is the Nakagami fading parameter, $\Omega$ the mean power, and $\rho$ is the normalized threshold level $(R / \sqrt{\Omega}) . \Gamma(a)$ and $\Gamma(a, b)$ denote the complete and incomplete Gamma functions, respectively. The average fade duration, $T_{R}$, of the diversity combined envelope is the total time spent below a threshold level $R$ divided by the number of individual fades and is estimated as

$$
T_{R}=\frac{C D F(r=R)}{N_{R}} .
$$

The normalized AFD $\left(T_{R}\right)$ formula for $M$-branch SC in a Nakagami fading environment is given by [15]

$$
T_{R} f_{m}=\frac{\Gamma\left(m, m \rho^{2}\right) \exp \left(m \rho^{2}\right)}{\sqrt{2 \pi} M\left(m \rho^{2}\right)^{m-0.5}} .
$$

The normalized LCR and AFD statistics for MRC [15] are accordingly listed in (8) and (9).

$$
\begin{aligned}
\frac{N_{R}}{f_{m}} & =\frac{\sqrt{2 \pi}}{\Gamma(m M)}\left(m \rho^{2}\right)^{m M-0.5} \exp \left(-m \rho^{2}\right) . \\
T_{R} f_{m} & =\frac{\Gamma\left(m M, m \rho^{2}\right) \exp \left(m \rho^{2}\right)}{\sqrt{2 \pi}\left(m \rho^{2}\right)^{m M-0.5}} .
\end{aligned}
$$

No closed-form expression exists for the solution of the $M$-branch EGC pdf. In [16], for i.i.d. channels the pdf of the EGC envelope $r$ is expressed as (10), at the bottom of the following page.

Under the assumption of branch envelopes with identical Nakagami parameters, and by restricting the number of available diversity branches to $M=2$, Iskander ([16, Eq. (38)]) reduced (10) to

$$
\begin{array}{r}
p(r)=\left(\frac{m}{\Omega}\right)^{2 m} \frac{2 B\left(2 m, \frac{1}{2}\right)}{[\Gamma(m)]^{2} 2^{2 m-2}} r^{4 m-1} \exp \left(-2 \frac{m}{\Omega} r^{2}\right) \\
\times \Phi\left(2 m, 2 m+\frac{1}{2}, \frac{m}{\Omega} r^{2}\right)
\end{array}
$$


where $B(a, b)$ is the beta function and $\Phi(a, b, c)$ is the confluent hypergeometric function. Following from this, the normalized LCR for dual-branch EGC is given as

$$
\begin{array}{r}
\frac{N_{R}}{f_{m}}=\frac{\sqrt{2 \pi} B\left(2 m, \frac{1}{2}\right)}{[\Gamma(m)]^{2} 2^{2 m-2}}\left(\frac{m}{\Omega} r^{2}\right)^{2 m-0.5} \exp \left(-2 \frac{m}{\Omega} r^{2}\right) \\
\times \Phi\left(2 m, 2 m+\frac{1}{2}, \frac{m}{\Omega} r^{2}\right) .
\end{array}
$$

Using the infinite series representation of the CDF for twobranch EGC, [16, Eq. (40)], allows the normalized AFD to be represented by

$T_{R} f_{m}=\frac{\exp \left(2 \frac{m}{\Omega} r^{2}\right) \sum_{n=0}^{\infty} \frac{\Gamma(2 m+n)}{\Gamma\left(2 m+n+\frac{1}{2}\right)} \frac{1}{2^{n} n !} \Gamma\left(2 m+n, 2 \frac{m}{\Omega} r^{2}\right)}{2 B\left(2 m, \frac{1}{2}\right)\left(2 \frac{m}{\Omega} r^{2}\right)^{2 m-0.5} \Phi\left(2 m, 2 m+\frac{1}{2}, \frac{m}{\Omega} r^{2}\right)}$.

Because of this limitation with the EGC pdf [(11)], the second-order statistical analysis and modeling presented here considers only two available signal branches (i.e. $M=2$ ), operating under the assumption of Nakagami fading conditions.

\section{B. Diversity Simulations}

In this study, we report the maximum likelihood estimated distributional parameters and the maximum Doppler frequency so that the small-scale fading experienced at the output of the hypothetical combiners considered here may be readily simulated. The waveforms may be straightforwardly generated using the following four-step process.

1) Generate $M$ random Nakagami sequences, corresponding to the required number of diversity branches, using the appropriate parameter estimates.

2) Combine the sequences using either (1), (2), or (3) depending on the desired combining scheme to achieve a signal waveform at the output of the combiner which closely approximates the desired distribution.

3) To introduce the correct autocorrelation, use a rank matching approach [19]. In this method, a Rayleigh sequence with the required autocorrelation function is generated using any commercially available software such as Matlab. Exploiting statistical rank, the Rayleigh sequence is then rearranged using order statistics, while keeping note of the original index of the data prior to rearrangement. Sort the combiner sequence generated from step 2 so that it is now also expressed in terms of order statistics (i.e., smallest to largest).

4) Now rearrange the simulated diversity combined sequence so that its autocorrelation structure matches closely that of the original (unordered) Rayleigh reference sequence using the indexes stored in step 3 .
TABLE IV

MEDian $\bar{m}$ AND $\bar{\Omega}$ PARAMETERS OVER All EXPERIMENTAL TRIALS

\begin{tabular}{|c|c|c|c|c|c|c|}
\hline & \multicolumn{2}{|c|}{ SC } & \multicolumn{2}{c|}{ EGC } & \multicolumn{2}{c|}{ MRC } \\
\hline Path & $\bar{m}$ & $\bar{\Omega}$ & $\bar{m}$ & $\bar{\Omega}$ & $\bar{m}$ & $\bar{\Omega}$ \\
\hline AB & 7.42 & 0.84 & 7.82 & 0.51 & 8.31 & 0.50 \\
\hline BA & 31.80 & 0.92 & 20.37 & 0.50 & 29.44 & 0.50 \\
\hline DC & 1.45 & 0.76 & 1.57 & 0.59 & 1.58 & 0.54 \\
\hline CD & 1.18 & 0.76 & 1.20 & 0.60 & 1.19 & 0.55 \\
\hline DE & 2.10 & 0.76 & 2.19 & 0.55 & 2.19 & 0.51 \\
\hline ED & 1.44 & 0.76 & 1.44 & 0.58 & 1.46 & 0.54 \\
\hline
\end{tabular}

This method of simulation has general applicability for diversity channel modelling, including scenarios outside of bodyworn communications.

\section{Second-Order Statistics}

To analyze the temporal distribution of small-scale fading at the output of the hypothetical combiners studied in Section IV, the combined data for each of the individual experimental trials was normalized to their respective local means prior to the parameter estimation process carried out below. Presenting the data in this manner has a number of distinct advantages in that the fading about the local mean signal level between combiners of the same type may be directly compared. Additionally, those wishing to simulate bodyworn diversity based on the results presented here may now assign their own mean signal level best suited to their practical application. Nakagami $m$ and $\Omega$ parameter values for each of the three combining schemes were estimated using maximum likelihood (ML) estimation. Due to the complexity of obtaining the derivative of the log-likelihood function for SC and EGC, the constrained minimization algorithm, fmincon, available in the optimization toolbox of Matlab, was used to perform ML estimation by minimizing the negative log-likelihood function over the bounded parameter space $\Theta$ (this procedure is equivalent to maximizing the log-likelihood).

Due to the large number of potential two-branch diversity combiners considered in this study, we summarize the parameter estimates for all two-branch combiners over each path in an identical manner to the analysis of the cross-correlation coefficient and diversity gain. Here $\bar{m}$ and $\bar{\Omega}$ are the mean parameter estimates of $m$ and $\Omega$, respectively for a particular combining scheme averaged over three repeated trials. The magnitude of $\bar{m}$ was found to be strongly influenced by the nature of the local surroundings (Table IV). For example, considering all two-branch receiver pairs using selection combining, the median parameter was found to decrease from $\bar{m}=7.42$ and $\bar{m}=31.80$ for paths $\mathrm{AB}$ and BA respectively in the anechoic chamber, to $\bar{m}=1.45$ for path $\mathrm{DC}$ and $\bar{m}=1.18$ for path $\mathrm{CD}$ in the open office area, with comparable reductions for paths

$$
p(r)=\sqrt{M} \int_{0}^{\sqrt{M} r} \int_{0}^{\sqrt{M} r-r_{M}} \ldots \int^{\sqrt{M} r-\sum_{i=3}^{M} r_{i}} \times\left[p_{1}(r)\left(r \sqrt{M}-\sum_{i=2}^{M} r_{i}\right) \prod_{i=2}^{M} p_{i}\left(r_{i}\right)\right] d r_{2} \ldots d r_{M} .
$$


TABLE V

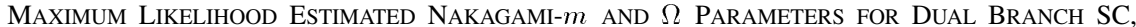
MRC AND EGC WhILE USER IS TRAVERSING CD IN OPEN OFFICE AREA

\begin{tabular}{|c|c|c|c|c|c|c|c|c|c|c|c|c|c|c|c|c|c|c|}
\hline & \multicolumn{6}{|c|}{$\mathrm{SC}$} & \multicolumn{6}{|c|}{ EGC } & \multicolumn{6}{|c|}{ MRC } \\
\hline Receivers & $\bar{m}$ & SE & $\bar{\Omega}$ & SE & $\overline{f_{m}}$ & SE & $\bar{m}$ & SE & $\bar{\Omega}$ & SE & $\bar{f}_{m}$ & SE & $\bar{m}$ & SE & $\bar{\Omega}$ & SE & $\bar{f}_{m}$ & SE \\
\hline $\begin{array}{l}\text { Back-Left / } \\
\text { Back-Right }\end{array}$ & 0.91 & 0.08 & 0.74 & 0.00 & 4.61 & 0.27 & 0.97 & 0.06 & 0.62 & 0.01 & 4.07 & 0.06 & 0.94 & 0.07 & 0.55 & 0.01 & 4.59 & 0.13 \\
\hline $\begin{array}{l}\text { Back-Left / } \\
\text { Left Abdomen }\end{array}$ & 0.91 & 0.02 & 0.74 & 0.00 & 3.76 & 0.21 & 1.06 & 0.01 & 0.62 & 0.00 & 4.30 & 0.21 & 0.98 & 0.01 & 0.56 & 0.00 & 4.36 & 020 \\
\hline $\begin{array}{l}\text { Back-Left / } \\
\text { Left-Chest }\end{array}$ & 1.06 & 0.01 & 0.75 & 0.01 & 4.50 & 0.21 & 121 & 0.05 & 0.60 & 0.01 & 4.19 & 0.14 & 1.14 & 0.03 & 0.54 & 0.01 & 4.23 & 0.10 \\
\hline $\begin{array}{l}\text { Back-Left / } \\
\text { Left-Wrist }\end{array}$ & 1.13 & 0.10 & 0.74 & 0.00 & 4.94 & 0.69 & 138 & 0.13 & 0.59 & 0.01 & 4.73 & 0.44 & 126 & 0.12 & 0.54 & 0.00 & 5.30 & 0.68 \\
\hline $\begin{array}{l}\text { Back-Left / } \\
\text { Right-Wrist }\end{array}$ & 0.97 & 0.07 & 0.73 & 0.01 & 4.42 & 0.30 & 1.13 & 0.09 & 0.60 & 0.02 & 4.32 & 0.24 & 1.06 & 0.08 & 0.54 & 0.01 & 4.89 & 029 \\
\hline $\begin{array}{l}\text { Back-Right / } \\
\text { Left-Abdomen }\end{array}$ & 1.18 & 0.01 & 0.76 & 0.01 & 3.82 & 0.12 & 1.15 & 0.07 & 0.63 & 0.02 & 3.92 & 0.09 & 1.17 & 0.04 & 0.56 & 0.01 & 4.06 & 0.05 \\
\hline $\begin{array}{l}\text { Back-Right / } \\
\text { Left-Chest }\end{array}$ & 1.57 & 0.03 & 0.77 & 0.01 & 4.74 & 0.08 & 1.48 & 0.05 & 0.58 & 0.00 & 4.27 & 0.01 & 1.54 & 0.05 & 0.54 & 0.00 & 4.32 & 0.04 \\
\hline $\begin{array}{l}\text { Back-Right / } \\
\text { Left-Wrist }\end{array}$ & 1.22 & 0.05 & 0.76 & 0.00 & 4.57 & 0.25 & 1.20 & 0.07 & 0.60 & 0.01 & 4.20 & 0.13 & 121 & 0.07 & 0.55 & 0.01 & 4.38 & 0.12 \\
\hline $\begin{array}{l}\text { Back-Right / } \\
\text { Right-Wrist }\end{array}$ & 1.23 & 0.10 & 0.76 & 0.01 & 4.46 & 0.30 & 1.17 & 0.08 & 0.63 & 0.02 & 3.80 & 0.14 & 1.19 & 0.10 & 0.56 & 0.01 & 4.15 & 0.18 \\
\hline $\begin{array}{l}\text { Left-Abdomen } \\
\text { / Left-Chest }\end{array}$ & 1.41 & 0.08 & 0.76 & 0.00 & 4.57 & 0.08 & 1.45 & 0.04 & 0.57 & 0.01 & 4.52 & 0.12 & 1.44 & 0.06 & 0.53 & 0.00 & 4.36 & 0.07 \\
\hline $\begin{array}{l}\text { Left-Abdomen } \\
\text { / Left-Wrist }\end{array}$ & 1.13 & 0.07 & 0.75 & 0.01 & 4.37 & 0.29 & 1.14 & 0.02 & 0.62 & 0.01 & 4.02 & 0.07 & 1.13 & 0.04 & 0.55 & 0.01 & 4.20 & 0.15 \\
\hline $\begin{array}{l}\text { Left-Abdomen } \\
\text { / Right-Wrist }\end{array}$ & 1.04 & 0.07 & 0.75 & 0.01 & 3.91 & 0.30 & 1.08 & 0.10 & 0.63 & 0.01 & 4.12 & 0.21 & 1.06 & 0.09 & 0.56 & 0.01 & 4.19 & 0.16 \\
\hline $\begin{array}{l}\text { Left-Chest / } \\
\text { Left-Wrist }\end{array}$ & 1.56 & 0.11 & 0.77 & 0.00 & 4.66 & 0.07 & 1.52 & 0.09 & 0.59 & 0.01 & 4.17 & 0.09 & 1.53 & 0.09 & 0.54 & 0.01 & 4.22 & 0.09 \\
\hline $\begin{array}{l}\text { Left-Chest / } \\
\text { Right-Wrist }\end{array}$ & 1.57 & 021 & 0.76 & 0.01 & 4.77 & 0.04 & 1.57 & 0.17 & 0.58 & 0.02 & 4.74 & 0.26 & 1.58 & 0.19 & 0.53 & 0.01 & 4.74 & 0.15 \\
\hline $\begin{array}{l}\text { Left-Wrist / } \\
\text { Right-Wrist }\end{array}$ & 1.26 & 0.07 & 0.76 & 0.02 & 4.49 & 0.16 & 1.43 & 0.07 & 0.60 & 0.01 & 4.47 & 0.09 & 136 & 0.07 & 0.55 & 0.01 & 4.51 & 0.14 \\
\hline
\end{tabular}

$\mathrm{DE}(\bar{m}=2.10)$ and $\mathrm{ED}(\bar{m}=1.44)$ in the hallway. This trend was also repeated for the EGC and MRC combining schemes as shown in Table IV. The large parameter estimates for $\bar{m}$ obtained in the anechoic chamber suggest that the signal which would be sampled at the output of these combiners operating in low multipath environments, such as outdoors, would undergo very little small-scale fading. In contrast, the lower $\bar{m}$ values obtained in the open office area and hallway $(\bar{m} \rightarrow 1)$, suggest that channel conditions encountered in these environments are close to those for an SC, EGC or MRC combiner operating in Rayleigh fading conditions.

Table V contains the estimated average Nakagami coefficients and maximum Doppler frequencies [1] for all of the virtual combiners considered while the user was traversing the path CD in the open office area. Also shown in Table V are the standard errors associated with the parameter estimates. In most cases, the standard errors were suitably low, providing evidence of a good degree of repeatability among experimental trials. This set of parameter estimates was chosen to demonstrate the typical small-scale fading characteristics, which could be measured by bodyworn diversity systems operating in a realistic indoor environment. From this set of results, two dual-branch combiners-back-right/left-chest and left-wrist/right-wrist-have been chosen to illustrate the modeling and simulation techniques presented above and to show the improvement in small-scale fading characteristics which may be obtained by designing a bodyworn system with more than one receive signal branch. Fig. 4 shows the theoretical and simulated LCRs and AFDs plots for the back-right/left-chest combination alongside the combined data from trial 3 . Also in- cluded for comparison are the LCR and AFD of the back-right signal branch, which was the receiver that recorded the signal envelope with the highest mean signal level. The parameter estimates for the theoretical plots and input to the simulator are those given in Table V. The simulated combiner output used for comparison consisted of 5000 samples. For all three combining schemes, it can be seen quite clearly that the crossing rates for the combined signal envelopes [Fig. 4(a), (c) and (e)] are significantly lower than if the bodyworn system consisted of only one receive path. In fact, all three schemes cross $10 \mathrm{~dB}$ below the local mean signal level at a rate of less than $0.3 \mathrm{~Hz}$, compared to approximately $2 \mathrm{~Hz}$ if the back-right receiver was considered in isolation. In Fig. 4(b), (d) and (f), the length of time spent by the combined signal envelope at lower signal levels is also reduced when compared to the back-right receiver.

Fig. 5 shows the LCR and AFD plots for the left-wrist/rightwrist combination while the user was moving along the path $\mathrm{CD}$ during trial 3 . The $\bar{m}$ parameters for this combination were lower than that of the previous example. A possible explanation for this was that both of the receive antennas in this virtual diversity system were positioned on the user's wrists which, due to the dynamic nature of these locations, would be expected to experience the significant signal variation. The $\overline{\rho_{c}}$ and $\overline{\text { DIMSL }}$ for left-wrist/right-wrist combination over path $\mathrm{CD}$ were -0.10 and $1.3 \mathrm{~dB}$ respectively, which was lower than that for the backright/left-chest pairing (Table III). Despite this, the $\overline{\mathrm{MRC}}$ diversity gain of $7.0 \mathrm{~dB}$ for the former was lower than backright/left-chest $(8.1 \mathrm{~dB})$ as shown in Table III. Finally, validation of the proposed diversity simulation process outlined above is also provided in Figs. 4 and 5 where the simulated, theoret- 

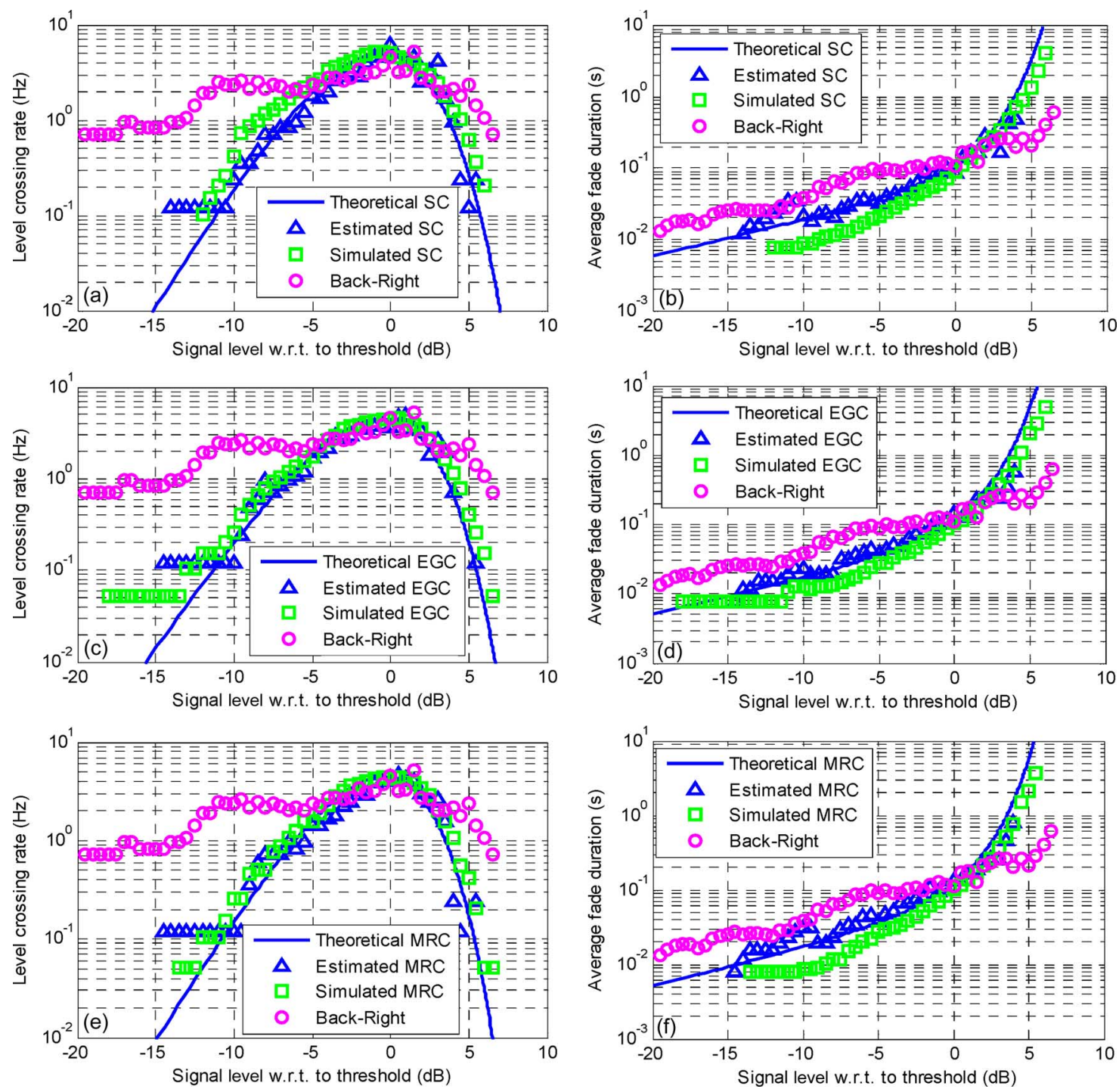

Fig. 4. Theoretical (continuous line), estimated (triangles) data from trial 3 and simulated (squares) two-branch (back-right/left-chest) diversity combining statistics for (a) SC, (b) EGC, and (c) MRC receivers operating in Nakagami fading conditions compared with branch with the highest mean (circles) for user following path $\mathrm{CD}$ in the open office area, all parameter estimates are provided in Table $\mathrm{V}$.

ical and empirical LCR and AFD plots are found to be in good agreement.

\section{CONCLUSION}

In this paper, cross-correlation coefficients between the signal fading experienced at various body locations covering the upper torso and arms of the human body have been reported. It was demonstrated that, for a mobile dual-branch bodyworn diversity system operating in either anechoic or indoor multipath conditions at $868 \mathrm{MHz}$, the cross-correlation coefficients between branches are typically less than 0.7 . The difference in mean signal level between branch signals was found to be dependent on the local environment, with a maximum $\overline{\text { DIMSL }}$ of $14.6 \mathrm{~dB}$ for the back-left/left-wrist receiver pairing when the test subject was moving away from the transmitter in the anechoic chamber. The corresponding maximum for the open office area, $6.3 \mathrm{~dB}$, occurred for the back-left/left abdomen receiver pair, while the test subject was also moving away from the transmitter. In a similar fashion to cross-correlation and DIMSL, diversity gain was also found to be greatly affected by the local surroundings and was generally observed to increase when the test subject moved from anechoic to multipath environmental conditions. With six upper body branches, fading beyond $3 \mathrm{~dB}$ below the mean signal level of the branch with highest mean power was eliminated when using either EGC or MRC. Furthermore, it was also shown that, in general, theoretical LCRs and AFDs for SC, MRC and EGC combiners operating in Nakagami fading conditions could be used to adequately describe second-order statistics calculated from measured data for bodyworn diversity. Based on ML estimation using combined signal envelopes, Nakagami- $m$ parameters were found to be greatest for diversity systems operating in anechoic conditions.

Finally, it is worth noting that while the experiments conducted in this study considered only one human test subject, it is anticipated that the variation of measured signal characteristics will be comparable for people with similar physique and agility while performing the same type of movements. Nonetheless, we would expect differences across the population as a whole. For 

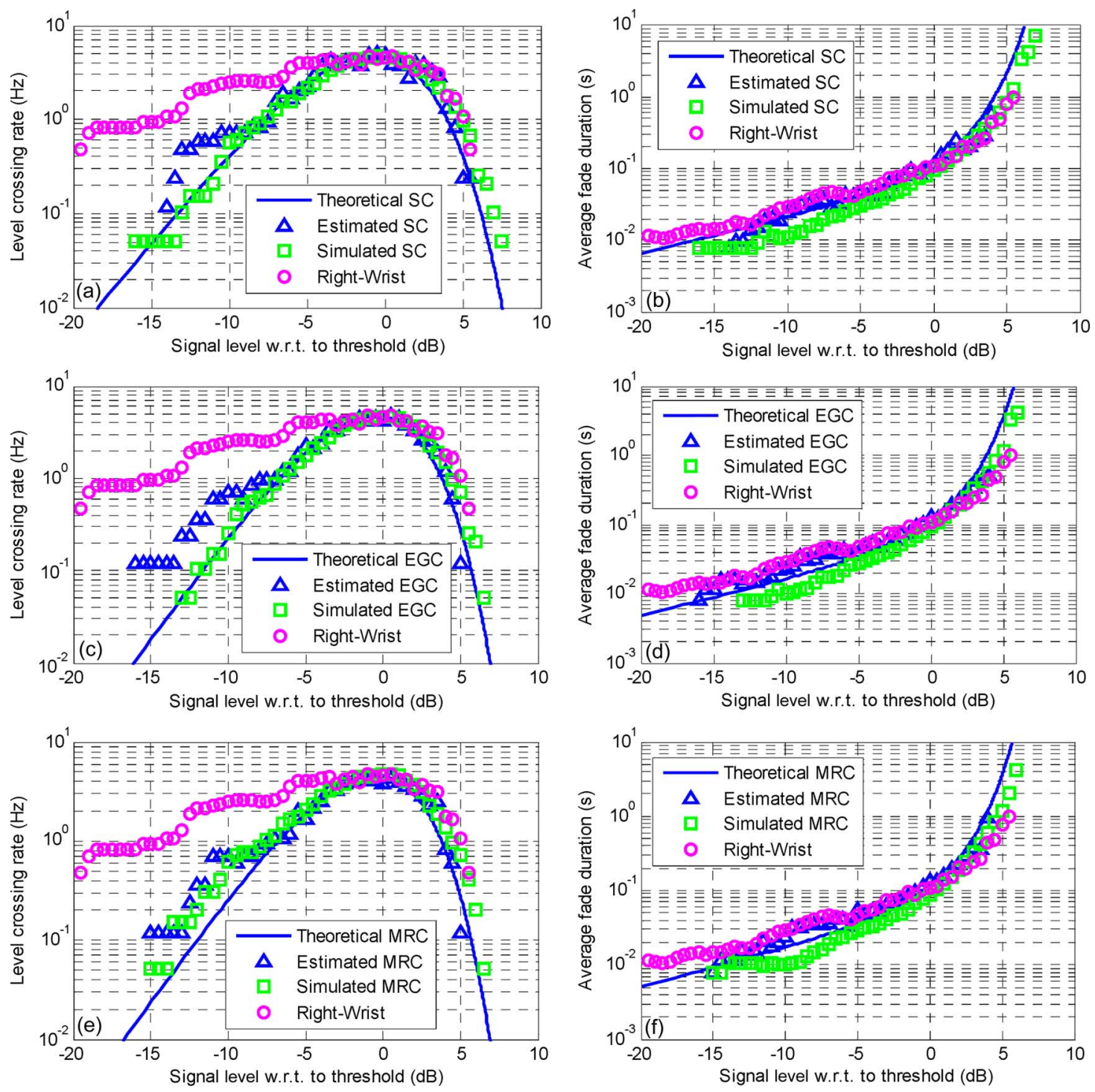

Fig. 5. Theoretical (continuous line), estimated (triangles) data from trial 3 and simulated (squares) dual-branch (left-wrist/right-wrist) diversity combining statistics for (a) SC, (b) EGC, and (c) MRC receivers operating in Nakagami fading conditions compared with branch with the highest mean (circles) for user following path $\mathrm{CD}$ in the open office area, all parameter estimates are provided in Table $\mathrm{V}$.

example, in [27] we observed noticeable variations in measured signal characteristics for uncontrolled experiments analyzing human body-to-body radio channel characteristics. Therefore, we recommend that further studies are conducted which take a cross-section of the population to specifically address the issue of the effect of variation in test subject upon off-body channel characteristics.

\section{ACKNOWLEDGMENT}

The authors would like to thank the anonymous reviewers whose constructive comments throughout the course of the review process have helped to significantly improve the quality and contribution of this work.

\section{REFERENCES}

[1] S. L. Cotton and W. G. Scanlon, "Characterization and modeling of the indoor radio channel at $868 \mathrm{MHz}$ for a mobile bodyworn wireless personal area network," IEEE Antennas Wireless Propag. Lett., vol. 6, pp. 51-55, 2007.
[2] H. R. Chuang, "Human operator coupling effects on radiation characteristics of a portable communication dipole antenna," IEEE Trans. Antennas Propag., vol. 42, pp. 556-560, Apr. 1994.

[3] W. G. Scanlon and N. E. Evans, "Numerical analysis of bodyworn UHF antenna systems," IEE Electron. Commun. Eng. J., vol. 13, no. 2, pp. 53-64, Apr. 2001.

[4] A. Molisch, Wireless Communications. Piscataway, NJ: IEEE Press, Wiley, 2005.

[5] N. Youssef, T. Munakata, and M. Takeda, "Fade statistics in Nakagami fading environments," in Proc. IEEE 4th Int. Symp. Spread Spectrum Tech. Applicat., Sep. 1996, vol. 3, pp. 1244-1247.

[6] S. L. Cotton and W. G. Scanlon, "A statistical analysis of indoor multipath fading for a narrowband wireless body area network," presented at the 17th IEEE Int. Symp. Personal, Indoor and Mobile Radio Commun. (PIMRC), Helsinki, Finland, Sep. 1-5, 2006.

[7] A. Fort, C. Desset, P. De Doncker, P. Wambacq, and L. Van Biesen, "An ultra-wideband body area propagation channel model-from statistics to implementation," IEEE Trans. Microw. Theory Technol., vol. 54, pp. 1820-1826, Jun. 2006.

[8] A. Fort, J. Ryckaert, C. Desset, P. De Doncker, P. Wambacq, and L. Van Biesen, "Ultra-wideband channel model for communication around the human body," IEEE J. Sel. Areas Commun., vol. 24, pp. 927-933, April 2006. 
[9] M. Nakagami, "The $m$-distribution: A general formula of intensity distribution of rapid fading," in Statistical Methods in Radio Wave Propagation. New York: Pergamon, 1960, pp. 3-36.

[10] N. C. Beaulieu and A. A. Abu-Dayya, "Analysis of equal gain diversity on Nakagami fading channels," IEEE Trans. Commun., vol. 39, pp. 225-234, Feb. 1991.

[11] E. Al-Hussaini and A. Al-Bassiouni, "Performance of MRC diversity systems for the detection of signals with Nakagami fading," IEEE Trans. Commun., vol. 33, pp. 1315-1319, Dece. 1985.

[12] P. R. Sahu and A. K. Chaturvedi, "Performance analysis of predetection EGC in exponentially correlated Nakagami- $m$ fading channel," IEEE Trans. Commun., vol. 53, pp. 1252-1256, Aug. 2005.

[13] O. C. Ugweje, "Selection diversity for wireless communications in Nakagami-fading with arbitrary parameters," IEEE Trans. Veh. Technol., vol. 50, pp. 1437-1448, No. 2001.

[14] S. O. Rice, "Statistical properties of a sine wave plus random noise," Bell Syst. Tech. J., vol. 27, pp. 109-157, Jan. 1948.

[15] M. D. Yacoub, C. R. C. M. da Silva, and J. E. V. Bautista, "Secondorder statistics for diversity-combining techniques in Nakagami-fading channels," IEEE Trans. Veh. Technol., vol. 50, pp. 1464-1470, Nov. 2001.

[16] C. D. Iskander and P. T. Mathiopoulos, "Analytical level crossing rates and average fade durations for diversity techniques in Nakagami fading channels," IEEE Trans. Commun., vol. 50, pp. 1301-1309, Aug. 2002.

[17] Q. T. Zhang, "A decomposition technique for efficient generation of correlated Nakagami fading channels," IEEE J. Sel. Areas Commun., vol. 18, pp. 2385-2392, Nov. 2000.

[18] N. C. Beaulieu and C. Cheng, "Efficient Nakagami-m fading channel simulation," IEEE Trans. Veh. Technol., vol. 54, pp. 413-424, Mar. 2005.

[19] J. C. S. S. Filho, M. D. Yacoub, and G. Fraidenraich, "A simple accurate method for generating autocorrelated Nakagami-m envelope sequences," IEEE Commun. Lett., vol. 11, pp. 231-233, Mar. 2007.

[20] C. D. Iskander and P. T. Mathiopoulos, "Fast simulation of diversity Nakagami fading channels using finite-state Markov models," IEEE Trans. Broadcast., vol. 49, pp. 269-277, Sep. 2003.

[21] E. C. Kohls, A. Abler, P. Siemsen, J. Hughes, R. Perez, and D. Widdoes, "A multi-band body-worn antenna vest," in Proc. IEEE Int. Symp. Antennas Propag. Society, Jun. 2004, vol. 1, pp. 447-450.

[22] U. Varshney and S. Sneha, "Patient monitoring using adhoc wireless networks: Reliability and power management," IEEE Commun. Mag., vol. 44, pp. 49-55, Apr. 2006

[23] S. L. Cotton and W. G. Scanlon, "Indoor channel characterization for a wearable antenna array at $868 \mathrm{MHz}$," in Proc. IEEE Wireless Commun. Network. Conf., Apr. 2006, vol. 4, pp. 1783-1788.

[24] S. L. Cotton and W. G. Scanlon, "Characterization and modeling of on-body spatial diversity within indoor environments at $868 \mathrm{MHz}$," IEEE Trans. Wireless Commun., vol. 8, no. 1, pp. 176-185, Jan. 2009.

[25] G. A. Conway, S. L. Cotton, and W. G. Scanlon, "An antennas and propagation approach to improving physical layer performance in wireless body area networks," IEEE J. Sel. Areas Commun., Special Issue Body Area Network.: Technol. Applicat., vol. 27, no. 1, pp. 27-36, Jan. 2009.

[26] I. Khan, P. S. Hall, A. A. Serra, A. R. Guraliuc, and P. Nepa, "Diversity performance analysis for on-body communication channels at 2.45 GHz," IEEE Trans. Antennas Propag., Special Issue Antennas Propag. Body-Centric Wireless Commun., vol. 57, no. 4, pp. 956-963, Apr. 2009.

[27] S. L. Cotton and W. G. Scanlon, "Channel characterization for single and multiple antenna wearable systems used for indoor body to body communications," IEEE Trans. Antennas Propag., Special Issue Antennas Propag. Body-Centric Wireless Commun., vol. 57, no. 4, pp. 980-990, Apr. 2009.
[28] Y. Wang, I. B. Bonev, J. O. Nielsen, I. Z. Kovacs, and G. F. Pedersen, "Characterization of the indoor multiantenna body-to-body radio channel," IEEE Trans. Antennas Propag., Special Issue Antennas Propag. Body-Centric Wireless Commun., vol. 57, no. 4, pp. 972-979, Apr. 2009.

[29] W. C. Jakes, Microwave Mobile Communications. New York: Wiley, 1974.

[30] D. G. Brennan, "Linear diversity combining techniques," Proc. IEEE, vol. 91, no. 2, pp. 331-356, Feb. 2003.

[31] A. M. D. Turkmani, A. A. Arowojolu, P. A. Jefford, and C. J. Kellett, "An experimental evaluation of the performance of two-branch space and polarization diversity schemes at $1800 \mathrm{MHz}$," IEEE Trans. Veh. Technol., vol. 44, pp. 318-326, May 1995.

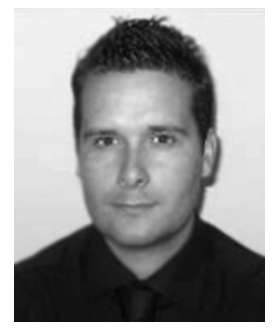

Simon L. Cotton (S'04-M'07) received the B.Eng. degree in electronics and software from the University of Ulster, Ulster, U.K., in 2004 and the Ph.D. degree in electrical and electronic engineering from the Queen's University of Belfast, Belfast, U.K., in 2007.

$\mathrm{He}$ is currently working as a Research Fellow with the Radio Communications Research Group of the Institute of Electronics, Communications and Information Technology (ECIT), Queen's University of Belfast, where he is investigating millimeter-wave technologies for personal communications and novel applications of short-range radio systems. His research interests include radio channel characterization and modeling for wireless body and personal area networks, measurements for transceiver diversity in bodyworn applications and simulation of wireless channels. He has authored and coauthored over 30 publications in major IEEE/IET journals and refereed international conferences.

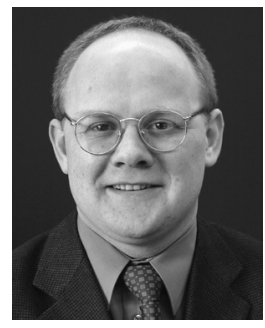

William G. Scanlon (M'98) received the B.Eng. degree in electrical engineering (first-class honors) by part-time study and the Ph.D. degree in electronics (specializing in wearable and implanted antennas) from the University of Ulster, Ulster, U.K., in 1994 and 1997, respectively.

He was appointed as Lecturer at the University of Ulster in 1998, then Senior Lecturer and Full Professor at Queen's University of Belfast, Belfast, U.K., in 2002 and 2008, respectively. He currently leads the Radio Communications research group at University of Belfast and he holds a part-time Chair in Short Range Radio at the University of Twente in the Netherlands. Prior to starting his academic career he had 10 years of industrial experience, having worked as a Senior RF Engineer for Nortel Networks, as a Project Engineer with Siemens and as a Lighting Engineer with GEC-Osram. His current research interests include personal communications, wearable antennas, RF and microwave propagation, channel modeling and characterization, wireless networking and protocols and wireless networked control systems. He has published over 150 research papers in major IEEE/IET journals and in refereed international conferences.

Prof. Scanlon received a Young Scientist award from URSI in 1999. He is a prolific reviewer for IEEE/IET journals and conferences and other major conferences. He is a member of the IEEE International Committee on Electromagnetic Safety (ICES) and the IASTED International Committee on Telecommunications. He served as a keynote speaker for the European Workshop on Conformal Antennas (2007). He Co-Chaired the 2009 Loughborough Antennas and Propagation Conference and he has acted as invited speaker and session chair at numerous other national and international conferences. 